\title{
El capital humano en los procesos de automatización: una primera aproximación al caso español
}

\author{
Álvaro Choi \\ Jorge Calero \\ Universidad de Barcelona e Instituto de Economía de Barcelona
}

\section{Resumen}

La robotización e introducción de inteligencia artificial en los procesos de producción plantean importantes retos en el mercado laboral. La literatura describe, entre otros efectos, la posibilidad de una destrucción de puestos de trabajo, parcial o totalmente compensada por la creación de nuevas ocupacones, así como una mayor demanda en la formación de los trabajadores. En este artículo se realiza una primera aproximación a esta cuestión para el caso español. Para ello, se analizan datos proveniente de la Encuesta de innovación de las empresas, elaborada por el Instituto Nacional de Estadística (INE), para el período comprendido entre los años 2006 y 2016, y se complementa con la explotación de datos de la Encuesta de Población Activa del INE. Los resultados confirman una mayor destrucción de empleo en las actividades con una mayor intensidad de innovación, así como un cambio en la demanda del perfil formativo de los trabajadores.

Palabras clave: automatización, robotización, inteligencia artificial, capital humano.

Clasificación JEL: J21, J23, J24, I24.

\begin{abstract}
Robotization and the introduction of artificial intelligence in the production processes pose important challanges in the labor market. Economic literature describes, among other effects, the possibility of job destruction, partially or totally offset by the creation of new occupations, as well as a greater demand in the training of workers. This article provides a first approach to this issue for the Spanish case. To do this, data from the National Institute of Statistics'(INE) Business Innovation Survey, are exploited, for the 2006-2016 period, and complemented by data from the Survey of Active Population of the INE. The results confirm a greater destruction of employment in innovation-intense activities, as well as a shift in the demand of the training profile of the workers.

Keywords: automation, robotization, artificial intelligence, human capital.
\end{abstract}

JEL clasiffication: J21, J23, J24, I24.

\section{Introducción}

La comprensión de los efectos socioeconómicos asociados a la introducción de innovaciones técnicas y nuevos sistemas de organización del trabajo ha despertado el interés de los economistas desde el siglo XVIII. Mientras que las revoluciones tecnológicas de los siglos XVIII y XIX se centraron principalmente en aumentar la 
productividad de trabajadores manuales poco cualificados, las innovaciones introducidas desde el siglo xx también han tendido a complementar la labor de trabajadores con cualificaciones media y alta (Gibbs, 2017). La distinta naturaleza de las innovaciones implica una fuerte limitación para la predicción de los efectos de las innovaciones contemporáneas (Mokyr, 2018). Por ejemplo, la introducción de la informática y digitalización ha llevado al surgimiento de fenómenos como la economía de plataformas, la transformación de buena parte de las ocupaciones y el auge del autoempleo individual (Eichhorst et al., 2017).

El desarrollo de la inteligencia artificial (IA) y los avances en robótica constituyen dos de los principales fenómenos incluidos en la denominada cuarta revolución industrial (o revolución 4.0) con importantes implicaciones sobre el nivel de ocupación y las características demandadas por el mercado laboral. Existe controversia acerca del impacto final de dichos fenómenos sobre el saldo neto de puestos de trabajo -número de nuevos puestos creados frente al número de antiguos puestos destruidos- (Arnzt et al., 2017). Mientras que autores como Frey y Osborne (2017) predicen una reducción en el número de puestos de trabajo disponibles, Autor y Salomons (2017) defienden lo contrario. Vivarelli (2014) matiza que las innovaciones en los procesos de producción tienden a ahorrar trabajo mientras que la innovación en productos tiende a aumentar su demanda. Por otro lado, sí parece existir cierto consenso acerca de las mayores y distintas competencias necesarias para poder desempeñar buena parte de los nuevos puestos de trabajo generados a partir de la automatización de los procesos productivos (Vivarelli, 2014; CEDEFOP, 2018). En su conjunto, la incertidumbre y riesgos que entraña la introducción de las innovaciones de la cuarta revolución industrial se han traducido en un incremento en la preocupación de los trabajadores (Dekker et al., 2017; Morikawa, 2017).

Este artículo realiza una primera aproximación a los efectos de los procesos de automatización del trabajo en el mercado de trabajo español, para el período 2006 a 2016. Más específicamente, se centra en el estudio de la evolución de los puestos de trabajo en las ocupaciones más propensas a ser automatizadas. Para ello, tras una revisión del estado de la cuestión, se realiza un análisis de la evolución del mercado laboral, a nivel agregado y por sectores, empleando microdatos de la Encuesta sobre Innovación de las Empresas del Instituto Nacional de Estadística (INE). Los resultados indican una caída en el empleo en las actividades con una mayor intensidad innovadora y un aumento en la demanda relativa de trabajadores más cualificados en dichas actividades y en otros sectores.

El artículo se estructura de la siguiente manera: el capítulo dos realiza una revisión de la literatura relativa a los efectos de la automatizaión en los mercados laborales; el capítulo tres describe, muy brevemente, la metodología y bases de datos empleados en el análisis, cuyos resultados se presentan y discuten en el capítulo cuatro; finalmente, el capítulo cinco sintetiza las principales conclusiones. 


\section{Automatización y capital humano: breve revisión de la literatura}

Este capítulo se estructura en dos apartados. En el primero (2.1) se describen los efectos de la automatización sobre el mercado laboral. El segundo apartado (2.2) hace lo propio para los efectos de un tipo específico de automatización, la inteligencia artificial (IA), sobre el mercado laboral.

\subsection{Robotización y mercado laboral}

La robotización o automatización consiste en la sustitución de trabajo por máquinas. A pesar de tratarse de un fenómeno iniciado con la primera revolución industrial, el proceso de robotización más reciente dispone de una serie de notas diferenciadoras: más allá de su mayor velocidad, se trata de máquinas que pueden realizar diversas tareas al mismo tiempo, más flexibles (que permiten superar la estandarización de los productos) y que pueden reajustarse por sí solas. Resultan especialmente susceptibles de ser automatizadas las tareas de tipo manual que consisten en la repetición rutinaria de operaciones. Pese a que en la actualidad existen pocos trabajos que puedan ser reemplazados completamente por máquinas, Boughin et al. (2017) exponen que, en EEUU, al menos el 60 por 100 de los puestos de trabajo incluyen al menos un 30 por 100 de actividades técnicamente automatizables.

A pesar de la novedad del fenómeno descrito, su relevancia ha llevado a que exista un nutrido grupo de estudios que analizan el efecto de la robotización sobre el mercado laboral, en general, y sobre sectores específicos. Un primer estudio que analiza el efecto agregado sobre el mercado laboral es el de Autor y Salomons (2017), quienes investigan si existe evidencia para sostener que los recientes procesos de robotización conducen a una reducción de la demanda agregada de trabajo y a un empeoramiento en las condiciones laborales (el denominado «robocalypse»). Exploran la relación, a nivel sectorial, entre el crecimiento en la productividad y el empleo en 19 países durante 35 años. De forma consistente con la narrativa del «robocalypse» y de la enfermedad de Baumol, observan que el empleo cae en la industria y sector agrario, al crecer la productividad. Sin embargo, simultáneamente demuestran que el nivel agregado de empleo crece, ya que los incrementos en la productividad aumentan los ingresos y el consumo. Las pérdidas de empleos en algunos sectores asociadas a aumentos en la productividad pueden quedar más que compensadas por los efectos colaterales positivos sobre el resto de la economía. Ahora bien, reconocen que los trabajadores del sector primario y secundario pueden encontrar dificultosa su incorporación al sector servicios, al demandar este último un nivel superior de cualificación. Nótese cómo lo anteriormente descrito implica una mayor polarización entre tipos de ocupaciones en el mercado laboral: mientras que los empleos poco cualificados se mantienen principalmente en el sector servicios -en tareas difícilmente automatizables-, el nivel de competencias requerido para el desempeño del resto de ocupaciones aumenta. 
Eichhorst (2015) discute a su vez la importancia de la velocidad de la implementación de los procesos de automatización. El proceso resultará menos disruptivo, tanto a nivel económico como político -tal y como advierte Levy (2018)-, si los actores disponen de tiempo para adaptarse y negociar soluciones. Pese a todo, el período de transición existirá, generándose desajustes temporales en el mercado.

Acemoglu y Restrepo (2017) analizan el efecto de la robotización en el sector industrial estadounidense entre 1990 y 2007. Para ello, tienen en cuenta el hecho de que la robotización, así como la externalización de procesos productivos rutinarios a terceros países, pueden generar polarización en los mercados laborales de los países desarrollados. Estiman un efecto negativo de los procesos de robotización sobre la ocupación y salarios. Este efecto resulta independiente del impacto de las importaciones desde países emergentes -cada producto incorpora un determinado nivel de tecnología-, la caída en los trabajos rutinarios, procesos de deslocalización, otros tipos de capital tecnológico y del stock total de capital. De acuerdo a sus estimaciones, un robot adicional por cada 1.000 trabajadores reduce la ratio de empleo sobre población entre 0,18 y 0,34 puntos porcentuales y los salarios, entre 0,25 y 0,5 puntos porcentuales. Estos impactos, no obstante, difieren por perfiles educativos, resultando mayores sobre los trabajadores con menores niveles educativos.

Dauth et al. (2017) alcanzan conclusiones similares a Acemoglu y Restrepo (2017) centrando su análisis en el impacto de la robotización sobre el empleo en el sector manufacturero alemán. No hallan un efecto negativo sobre el nivel de empleo, pero sí un cambio en su composición. Estiman que, si bien cada robot destruyó dos puestos de trabajo en el sector manufacturero entre 1994 y 2014, estos quedaron más que compensados por el aumento en la ocupación en el sector servicios. La caída en los empleos en el sector manufacturero alemán viene explicada por la reducción en la contratación de trabajadores jóvenes, observándose, a su vez, la creación de un mercado de trabajo con insiders y outsiders, siendo los primeros aquellos trabajadores empleados en empresas manufactureras que han introducido robots en sus procesos de producción. Los insiders tienden a tener un trabajo más estable, si bien ello no implica que siempre desarrollen las mismas labores en el seno de esa empresa, tras la introducción de robots. Ahora bien, la mayor estabilidad en el empleo de los insiders tiene como contrapartida un menor nivel salarial para los que tienen un nivel formativo medio o bajo. Este efecto negativo sobre los salarios no se observa, sin embargo, para trabajadores más cualificados. En una línea similar, estudios como Hutter y Weber (2017) o Caines et al. (2017) concluyen que los trabajadores con un reducido nivel de competencias para resolver tareas complejas son los más desprotegidos ante los efectos de los procesos de automatización. Schmidtpeter y Winter-Ebmer (2018) proponen las políticas activas de empleo como un método efectivo para facilitar la reincorporación al mercado laboral de los desempleados por motivos tecnológicos.

Decker et al. (2017), por su parte, analizan el efecto de la robotización en un sector menos expuesto, hasta el momento, a la automatización: los servicios. Argumentan que los reducidos salarios de los trabajadores menos cualificados del sector servicios harán poco rentable su sustitución por robots. Vaticinan que resultará más 
probable la robotización de servicios para los que se requiera una cualificación media. El hecho de que los robots modernos sean mucho más seguros que los de generaciones anteriores, así como el desarrollo de software y hardware de uso intuitivo, facilita la complementariedad del trabajo entre robots y humanos, incluso para personas poco cualificadas. Los servicios con aplicaciones de robótica más prometedoras son, según Decker et al. (2017), aquellos que implican trabajo nocturno, esfuerzo físico y problemas de salud.

Sachs et al. (2015) abordan los efectos redistributivos de la robotización y defienden la introducción de políticas redistributivas capaces de compensar los efectos potencialmente negativos que puede tener la robotización sobre el bienestar social. Exponen que estos efectos serán especialmente negativos para los trabajadores jóvenes y de las generaciones futuras si los bienes que pueden producirse con o sin robots son altamente sustituibles y en aquellas actividades en las que los robots pueden sustituir fácilmente al trabajo humano.

Siguiendo con los efectos redistributivos de la robotización, Berg et al. (2018), mediante un modelo de equilibrio general, llegan a la conclusión de que la robotización tiene un efecto positivo sobre el crecimiento económico pero incrementa la desigualdad económica, ya que los salarios de buena parte de la población se reducen. Consideran demasiado optimistas los estudios existentes que afirman que, mientras el nivel de competencias de los trabajadores se vaya actualizando, el efecto sobre el volumen total de puestos de trabajo y sobre los salarios será reducido. Berg et al. (2018) demuestran que, cuanto mayor sea la capacidad de sustituir máquinas por robots, mayor será la caída en los salarios y mayor el aumento en la retribución al capital, al menos en el corto plazo -corto plazo que consideran que puede consistir en varias generaciones. En estas circunstancias, los salarios solo se recuperarían en el momento en el que el volumen de capital acumulado resulte muy elevado $-\mathrm{y}$, por tanto, el capital humano pase a ser escaso, en términos relativos.

Caselli y Manning (2018), por el contrario, desarrollan un modelo en el que muestran que la nueva tecnología no tiene por qué tener necesariamente un efecto depresor sobre los salarios de los trabajadores. Los salarios de hecho subirán si el precio de los bienes de inversión cae más que el de los bienes de consumo. Sí alertan, no obstante, acerca de que la amenaza para los salarios asociada a la introducción de nuevas tecnologías puede venir de la apertura al mercado internacional.

Finalmente, y a diferencia de los estudios anteriores, Maloney y Molina (2016) analizan los efectos de la automatización sobre los países en vías de desarrollo, subrayando que los efectos de la cuarta revolución para estos no tienen que coincidir con los de los países desarrollados. Muestran que la automatización y externalización a terceros países de las actividades más rutinarias genera polarización en los mercados laborales de los países desarrollados, mientras que los datos no permiten afirmar lo mismo para los países en vías de desarrollo en la actualidad. Cabe la posibilidad, no obstante, de que en el futuro cercano sí aumente la polarización en los mercados laborales de los países en vías de desarrollo si estos externalizan a su vez su producción e incorporan rápidamente robots a sus procesos de producción. 


\subsection{Inteligencia artificial y mercado laboral}

La IA es la capacidad de las máquinas para simular capacidades cognitivas similares a los humanos; es decir, la capacidad de las computadoras para tomar decisiones, resolver problemas y acumular conocimiento. En otras palabras, se trata de software «inteligente». Llevada a su extremo, la IA debería permitir a las máquinas desarrollar su propio «aprendizaje» de forma autónoma, desarrollándose una singularidad (aquella situación en la que un dispositivo con IA consigue ser capaz de desarrollarse a sí mismo). La IA constituye una de las formas más avanzadas de automatización y, tal y como exponen Brynjolfsson et al. (2017), su implementación y efectos se encuentran todavía en una fase muy incipiente. En la actualidad, las actividades con un mayor grado de penetración de la IA son, además de la automoción, las telecomunicaciones y las finanzas (Bughin et al., 2017).

Como se podrá intuir, la IA tiene implicaciones distintas a las de la robotización sobre el mercado de trabajo. Varian (2018) expone que la IA puede incidir sobre los rendimientos a escala de tres posibles maneras: por el lado de la oferta, reduciendo costes medios; por el lado de la demanda, a través de efectos de red (el valor del bien también depende del número de personas que adquieran ese bien -tal y como sucede, por ejemplo, con sistemas operativos y aplicaciones informáticas-); y, finalmente, vía learning by doing, si bien esta fuente de mejora de la productividad depende especialmente de las competencias y habilidades de los trabajadores.

Los estudios que analizan los efectos de la IA sobre los mercados laborales y la demanda laboral tienden a basarse en modelos, dada la escasez de datos. Tal y como exponen Raj y Seamans (2018), demostrar la complementariedad o sustituibilidad entre IA, robots y trabajadores requeriría el uso de información muy detallada a nivel de empresa. Arnzt et al. (2017) muestran que, en efecto, los análisis a nivel de sector tienden a sobreestimar el efecto de la automatización sobre el empleo, en comparación con los resultados obtenidos utilizando datos a nivel de trabajador. Una posible explicación es el hecho de que solo algunos trabajadores se especializan en una serie de tareas que complementan a robots e IA.

Los resultados de los modelos que estiman los efectos de la IA dependen, en buena medida, de la combinación de los siguientes factores, señalados por Brynjolfsson y Mitchell (2017): la facilidad técnica en la sustitución de puestos de trabajo por IA; la elasticidad precio de las distintas tareas a sustituir por IA, así como su efecto sobre los precios de los bienes y servicios producidos; la complementaredad de la IA con ciertos tipos de habilidades y competencias humanas; la elasticidad renta de los bienes, al modificarse los ingresos de toda o parte de la población; la elasticidad de la oferta laboral a cambios en la demanda laboral; y el rediseño de los procesos de producción.

Aghion et al. (2017) modelizan los efectos de la IA sobre el crecimiento económico y la distribución de las rentas entre trabajo y capital. Estiman que la enfermedad de Baumol limita la posibilidad de automatización de toda la economía, al existir tareas de difícil automatización -sobre todo en los servicios-. Sí consideran, en 
todo caso, que la introducción de IA hace que las economías en su conjunto aumenten los requisitos de competencias de sus trabajadores. Sin embargo, las empresas más intensivas en el uso de IA tendrán una mayor tendencia a externalizar las tareas que requieran una baja cualificación y pagar un mayor salario a los trabajadores con menores niveles de cualificación que permanezcan en su seno (de la propia empresa). Cowen (2018), no obstante, argumenta que en un entorno con capital humano abundante, los beneficios monetarios de la educación pueden ser bajos.

Morikawa (2017), por su parte, emplea datos japoneses para analizar el efecto de la IA y robots sobre el nivel de empleo y las características demandadas a los trabajadores. Los resultados apuntan a la importancia de que los trabajadores acumulen competencias flexibles a través de la educación superior, especialmente en las áreas de ciencias e ingeniería. Señala a su vez la mayor dificultad para sustituir con IA y robots las competencias específicas de determinados puestos de trabajo. Los servicios personales más intensivos en el uso de capital humano son las actividades económicas menos propensas a ser sustituidas por IA y robots por dos motivos: en primer lugar, la dificultad técnica para sustituir la actividad humana por máquinas; en segundo lugar, la preferencia de los consumidores a ser atendidos por humanos en estas actividades.

Korinek y Stiglitz (2017) y Cowen (2018) discuten una de las potenciales consecuencias de la implementación de la IA y otros avances teconológicos con capacidad para sustituir el trabajo humano: los efectos redistributivos. Korinek y Stiglitz (2017) identifican dos canales a través de los cuales la introducción de IA puede afectar a la desigualdad, más allá de la destrucción de puestos de trabajo y las reducciones salariales: el excedente del que se apropian los innovadores y la redistribución asociada a los cambios en el precio de los factores. Cowen (2018) describe que parte de los efectos negativos asociados a la caída de los salarios puede ser compensada por rebajas en los precios de los bienes de consumo al reducirse los costes de producción. En bienes como las redes sociales, el coste marginal puede llegar a ser cero. Ello amortiguaría los efectos redistributivos asociados a la reducción en el número de puestos de trabajo y en los salarios.

Korinek y Stiglitz (2017) defienden que la redistribución vía impuestos y prestaciones compensatorias puede ser un mecanismo óptimo para compensar la caída de ingresos de los perdedores del proceso de implementación de IA. Ello facilitaría a su vez la introducción de la IA, al reducirse la resistencia al cambio -que podría frenar el crecimiento económico- por parte de los trabajadores amenazados por el cambio tecnológico. Consideran, por tanto, que cuanto mayor sea el apoyo vía políticas compensatorias, más rápida podrá ser la transición hacia el nuevo paradigma tecnológico y menores los costes sociales. Cowen (2018), sin embargo, se muestra escéptico respecto a la efectividad de las políticas redistributivas para paliar los efectos de la automatización.

A nivel internacional, la introducción de IA en los países desarrollados puede llevar a una reversión en los procesos de deslocalización, siempre que la caída en los niveles salariales en aquellos sea lo suficientemente elevada (Cowen, 2018). Nótese 
que ello podría dificultar el desarrollo de los países más pobres, que no podrían competir vía diferencias en los salarios. Finalmente, más allá de los efectos vía precios relativos vinculados al comercio internacional, Cowen (2018) explica que, a nivel global, cuanto más concentrada esté la producción de un bien en una zona, mayor será el efecto positivo de la IA sobre los consumidores globales.

La revisión realizada permite plantear las siguientes dos hipótesis, para el caso español: en primer lugar, la robotización e IA conducirán a la caída en la ocupación en los sectores más expuestos a la automatización; en segundo lugar, la automatización conduce a un incremento en la demanda de trabajadores más formados. Dado el carácter exploratorio del estudio, no se analizan los efectos sobre los niveles salariales ni la evolución de los precios relativos de los bienes y servicios.

\section{Metodología y datos}

Presentamos a continuación un análisis de las relaciones entre los procesos de innovación, los ámbitos de actividad en las empresas, y el capital humano de los trabajadores que se ven afectados por tales procesos de innovación.

Para conocer el nivel de innovación en cada rama de actividad empresarial, utilizaremos datos de la Encuesta sobre la innovación en las empresas, del INE. Esta encuesta tiene como ámbito poblacional todas las empresas agrícolas, industriales, de construcción y de servicios con al menos diez personas ocupadas remuneradas. Hemos utilizado datos correspondientes a las ediciones comprendidas entre 2006 y 2016. La muestra utilizada en la edición de 2016 fue de 38.742 empresas. En esta encuesta, la innovación tecnológica se define del siguiente modo: «Las innovaciones tecnológicas comprenden los productos (bienes o servicios) y procesos tecnológicamente nuevos, así como las mejoras tecnológicas importantes de los mismos. Una innovación tecnológica se considera como tal cuando se ha introducido en el mercado (innovaciones de productos) o se han utilizado en el proceso de producción de bienes o de prestación de servicios (innovaciones de proceso)». Se trata, por tanto, del tipo de innovación relevante para nuestro análisis. La encuesta ofrece, también, datos diferenciados relativos a la innovación no tecnológica, relacionada con cambios en el marketing o en la organización.

La variable que hemos seleccionado como indicador de la actividad innovadora es la «intensidad de la innovación tecnológica», que se mide por el INE como la proporción que suponen los gastos en actividades de innovación tecnológica sobre la cifra de negocios (en porcentaje). Este indicador es utilizado aquí como una proxy del nivel de automatización.

Adicionalmente, la información referida a los niveles de ocupación en cada ámbito de actividad empresarial y su evolución a lo largo del tiempo ha sido extraída de los datos de la European Labour Force Survey, de EUROSTAT (consulta online) y de la Encuesta de Población Activa (a través de los microdatos de los segundos trimestres de 2007 y 2018). 


\section{Resultados y discusión}

En el Gráfico 1 presentamos una primera aproximación a la evolución de la innovación tecnológica en España. Se observa una cierta estabilidad en la intensidad de la innovación tecnológica lo largo del periodo de tiempo considerado (2006 a 2016), con una ligera subida en 2009 y una reducción posterior en 2010 y 2011. La intensidad es mayor en el sector industrial y menor en el sector primario donde, además, se produce una caída considerable entre 2009 y 2010.

Con objeto de conocer las relaciones entre ramas de actividad (en función de su intensidad de innovación) y los procesos de creación y destrucción de empleo, hemos establecido tres grupos de ramas de actividad. El grupo de actividades de innovación tecnológica baja incluye aquellas ramas con un índice inferior a 0,4 (insistimos en que el indicador se refiere al porcentaje del gasto en innovación tecnológica sobre la cifra de negocios); el grupo de intensidad media engloba las ramas con un índice comprendido entre 0,4 y 1 , mientras que en el grupo de intensidad alta aparecen todas las ramas con índices superiores a 1. En la Tabla A.1 del Anexo aparece el detalle de esta agrupación.

En el Gráfico 2 se expone la evolución de la intensidad tecnológica para una serie de ramas de actividad de alta intesidad de innovación, como son las ramas de Farmacia, Productos informáticos, electrónicos y ópticos, Vehículos de motor y Actividades profesionales, científicas y técnicas; en todos los casos la intensidad de la innovación es muy alta, superior al 2 por 100 de la cifra de negocios y llegando a

\section{GRÁFICO 1}

\section{EVOLUCIÓN DE LA INTENSIDAD DE INNOVACIÓN TECNOLÓGICA POR RAMAS DE ACTIVIDAD. ESPAÑA}

(En \%)

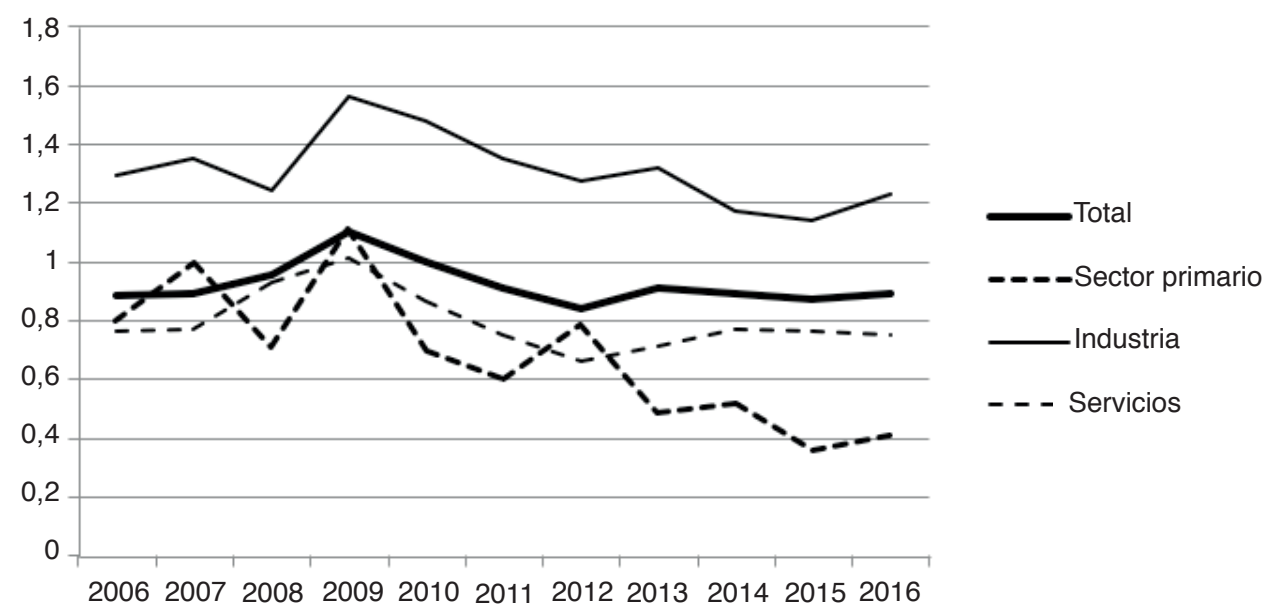

FUENTE: Elaborado a partir de Encuesta sobre la innovación en las empresas, INE. 


\section{GRÁFICO 2}

EVOLUCIÓN DE LA INTENSIDAD DE INNOVACIÓN TECNOLÓGICA POR RAMAS DE ACTIVIDAD. RAMAS DE ELEVADA INTENSIDAD DE INNOVACIÓN. ESPAÑA

(En \%)

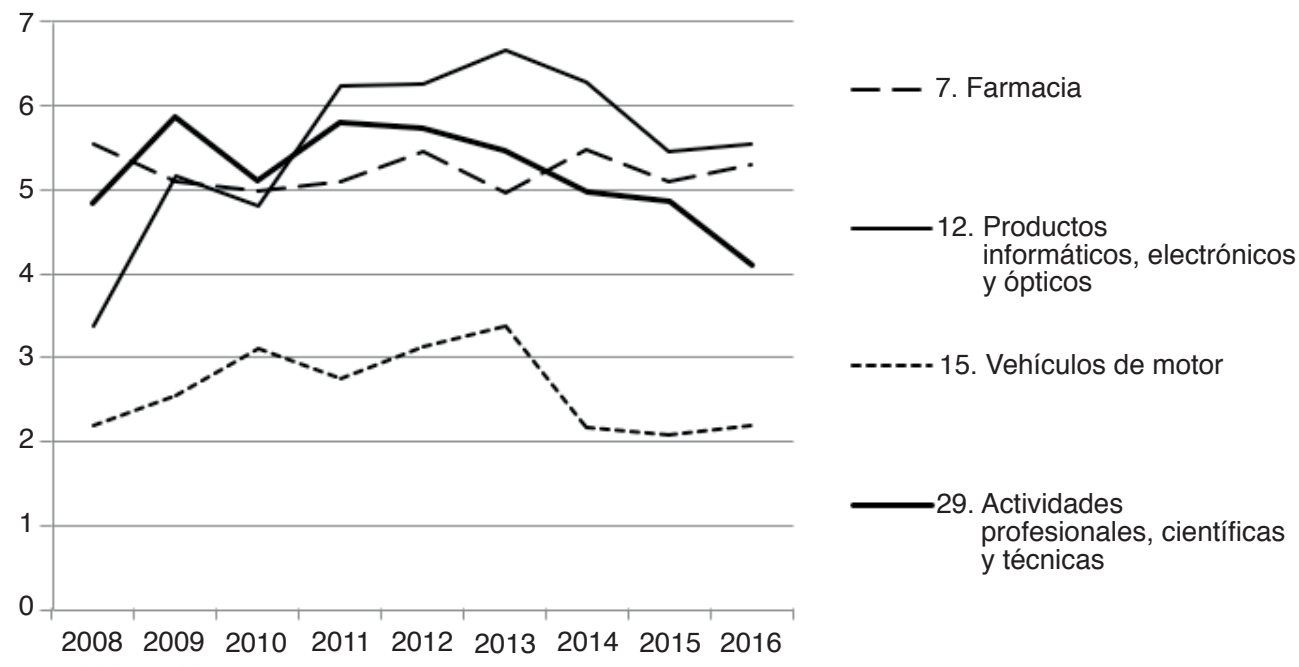

FUENTE: Elaborado a partir de Encuesta sobre la innovación en las empresas, INE.

más del 6 por 100 en algunas de ellas. En la mayor parte de las ramas el nivel de intensidad de innovación tecnológica es similar al principio y al final del periodo. Por el contrario, en el Gráfico 3, correspondiente a ramas de actividad de baja intensidad de innovación tecnológica, la tendencia ha sido decreciente. En las cuatro ramas seleccionadas como ejemplo, Energía y agua, Construcción, Comercio y Actividades administrativas y servicios auxiliares, la intensidad de la innovación tecnológica es más baja en 2016 que diez años antes.

Veamos, a continuación, cómo han evolucionado los niveles de ocupación en los tres grupos que hemos establecido. En el Gráfico 4 aparece la evolución, desde 2008 hasta 2017, para el caso español, del número de trabajadores ocupados. Se recoge el fuerte impacto de la crisis, que provoca una pérdida de empleos en los tres grupos hasta el año 2013 y una posterior recuperación. El grupo en el que más rápidamente se pierde empleo, entre 2008 y 2011, es el de alta intensidad de innovación tecnológica. La pérdida de empleos en el grupo de baja intensidad de innovación tecnológica es solo ligeramente menor. La recuperación, a partir de 2014, es paralela en los grupos de baja y alta intensidad de innovación tecnológica. Ambos grupos han perdido más empleos, durante todo el periodo, que el grupo de media intensidad de innovación. Según estos datos, la pérdida de empleos rápida durante la crisis puede estar relacionada con una tasa de innovación tecnológica elevada, que permite una mayor rapidez en la sustitución de empleo por tecnología cuando las condiciones de 


\section{GRÁFICO 3}

EVOLUCIÓN DE LA INTENSIDAD DE INNOVACIÓN TECNOLÓGICA POR RAMAS DE ACTIVIDAD. RAMAS DE BAJA INTENSIDAD DE INNOVACIÓN. ESPAÑA

(En \%)

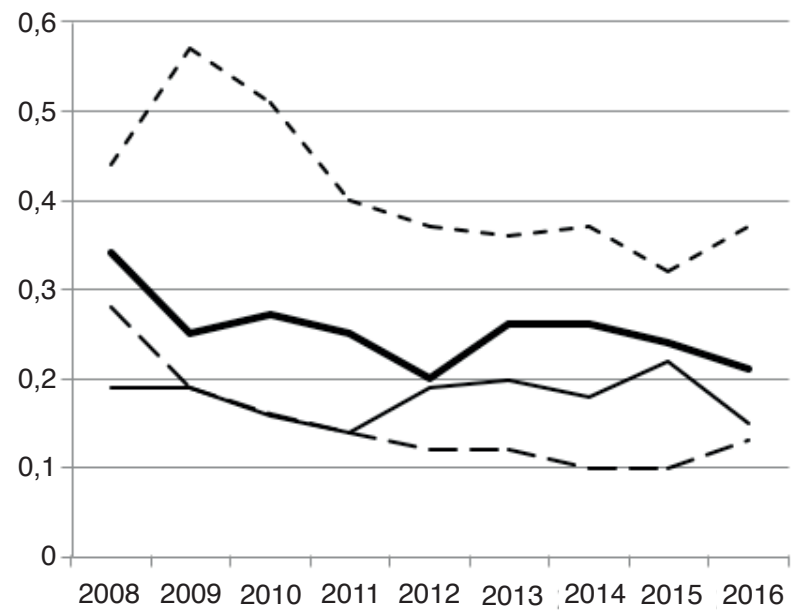

- - 20. Energía y agua

22. Construcción

- 23. Comercio

-30. Actividades administrativas y servicios auxiliares

FUENTE: Elaborado a partir de Encuesta sobre la innovación en las empresas, INE.

\section{GRÁFICO 4}

EVOLUCIÓN DEL NÚMERO DE OCUPADOS EN FUNCIÓN DE LA INTENSIDAD DE INNOVACIÓN TECNOLÓGICA DE LA RAMA DE ACTIVIDAD.

ESPAÑA

$(2008=100)$

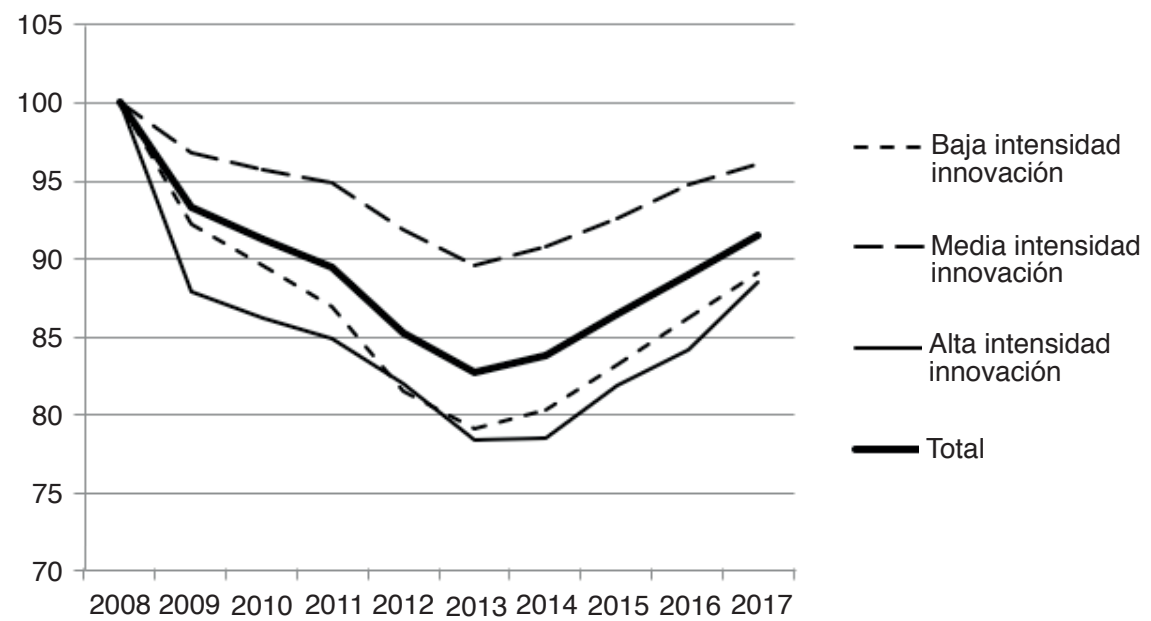

FUENTE: Elaborado a partir de Encuesta sobre la innovación en las empresas, INE y de European Labour Force Survey, EUROSTAT. 
mercado exigen reducir costes. Pero, también, con una tasa de innovación reducida, que se corresponde con la existente en ramas de actividad especialmente castigadas por la crisis, como es la construcción. Por el contrario, el grupo correspondiente a una intensidad media de innovación tecnológica presenta pérdidas de empleo más moderadas (10 por 100 entre 2008 y 2013 y únicamente un 4 por 100 entre 2008 y 2017).

Los Gráficos 5 y 6 permiten establecer una comparación entre el caso español y el de otros países de nuestro entorno ${ }^{1}$. En el Gráfico 5, referido a los 28 países de la Unión Europea, se observa, como es lógico, un efecto mucho más limitado de la crisis sobre la pérdida de los empleos (en 2016 ya se recuperó, en el conjunto de la Unión Europea, el nivel de empleo previo a la crisis). Pero también observamos, igual que sucede en España, que la pérdida más rápida de empleo se produce en el grupo de actividades de mayor intensidad de innovación tecnológica. También, en el conjunto de países europeos, el nivel de empleo en este grupo al final del periodo (2017) es prácticamente idéntico que al principio del mismo, mientras que en los otros grupos se ha producido una mayor creación de empleo.

El Gráfico 6 presenta los datos correspondientes al caso de Alemania, país clasificado como ejemplo de país líder en innovación según los criterios del European Innovation Scoreboard (Comisión Europea, 2017). El efecto de la crisis en Alemania es mucho más limitado que en España y, también, que en la mayor parte del resto de países de la Unión Europea (de hecho, en 2017 el nivel de empleo es un 7 por 100 mayor que en 2008). Vemos, también en este caso, que el grupo de alta intensidad de innovación tecnológica pierde más rápidamente empleo que la media entre 2008 y 2010, y al final del periodo tiene un nivel de empleo algo más bajo, en términos relativos, que la media. En el caso alemán, a diferencia de los anteriores, el empleo en el grupo de baja intensidad de innovación crece más rápidamente que en el resto de grupos. Recuérdese, al respecto, que el grupo de baja intensidad de innovación tecnológica incluye actividades muy dispares entre sí.

Finalizaremos este apartado describiendo los cambios en la composición de la población ocupada según su nivel de capital humano y las ramas de actividad del empleo. En el Cuadro 1 podemos observar la evolución, entre 2007 y 2018, de la composición interna del empleo en España según su cualificación, para diferentes ramas de actividad (la Tabla A.2 del Anexo presenta los valores de la composición interna en el año inicial, 2007). Los valores negativos indican una pérdida de empleos correspondientes al nivel educativo determinado, mientras que los valores positivos implican ganancias de empleos. En conjunto, durante el periodo se ha producido un trasvase desde el empleo menos cualificado hacia el empleo más cualificado, de en torno a diez puntos porcentuales. Las ramas de actividad donde este trasvase ha sido

${ }^{1}$ Es preciso tener en cuenta, como limitación a este procedimiento, que los grupos de actividades en función del nivel de innovación tecnológica que aplicamos aquí son los obtenidos a partir de la Encuesta sobre la innovación en las empresas española. Esta agrupación no tiene por qué ser generalizable a otros países europeos, donde el nivel de innovación tecnológica puede ser diferente en diversas ramas de actividad. 


\section{GRÁFICO 5}

\section{EVOLUCIÓN DEL NÚMERO DE OCUPADOS EN FUNCIÓN DE LA INTENSIDAD} DE INNOVACIÓN TECNOLÓGICA DE LA RAMA DE ACTIVIDAD.

\section{UNIÓN EUROPEA}

$(2008=100)$

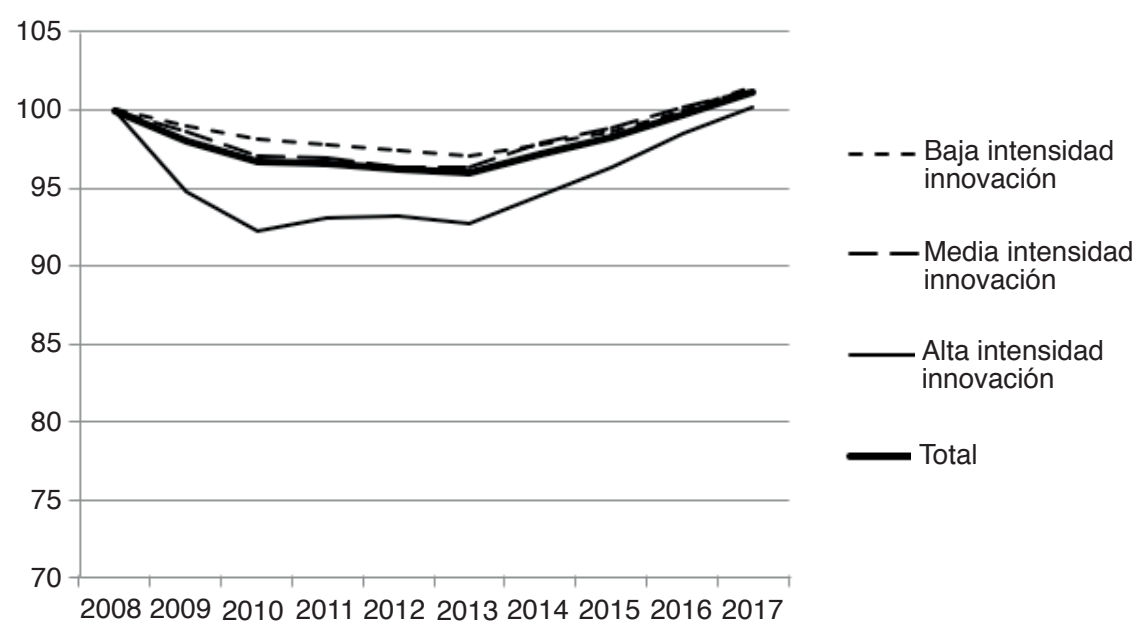

FUENTE: Elaborado a partir de Encuesta sobre la innovación en las empresas, INE y de European Labour Force Survey, EUROSTAT.

\section{GRÁFICO 6}

EVOLUCIÓN DEL NÚMERO DE OCUPADOS EN FUNCIÓN DE LA INTENSIDAD DE INNOVACIÓN TECNOLÓGICA DE LA RAMA DE ACTIVIDAD. ALEMANIA

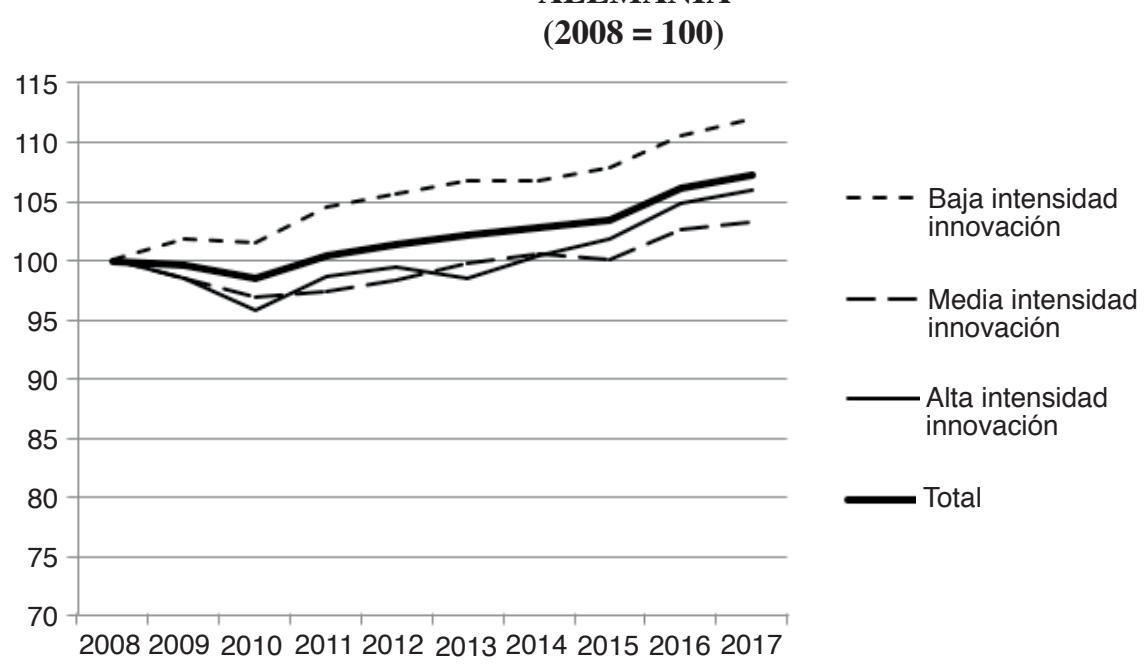

FUENTE: Elaborado a partir de Encuesta sobre la innovación en las empresas, INE y de European Labour Force Survey, EUROSTAT. 


\section{CUADRO 1}

VARIACIONES EN LA DISTRIBUCIÓN DE LOS OCUPADOS SEGÚN SU NIVEL EDUCATIVO Y SEGÚN RAMA DE ACTIVIDAD. COMPARACIÓN ENTRE 2018 (SEGUNDO TRIMESTRE) Y 2007 (SEGUNDO TRIMESTRE). ESPAÑA (En puntos porcentuales)

\begin{tabular}{|c|c|c|c|}
\hline & $\begin{array}{c}\text { Hasta } \\
\text { secundaria } \\
\text { inferior }\end{array}$ & $\begin{array}{c}\text { Secundaria } \\
\text { superior }\end{array}$ & Superior \\
\hline Agricultura, ganadería, silvicultura y pesca & $-2,27$ & 1,36 & 0,91 \\
\hline $\begin{array}{l}\text { Industria de la alimentación, textil, cuero, madera y } \\
\text { papel }\end{array}$ & $-4,34$ & $-0,39$ & 4,73 \\
\hline $\begin{array}{l}\text { Industrias extractivas, refino de petróleo, industria } \\
\text { química, farmacéutica, industria del caucho y mate- } \\
\text { rias plásticas, suministro energía eléctrica, gas, vapor } \\
\text { y aire acondicionado, suministro de agua, gestión de } \\
\text { residuos. Metalurgia }\end{array}$ & $-9,55$ & $-0,87$ & 10,42 \\
\hline $\begin{array}{l}\text { Construcción de maquinaria, equipo eléctrico y mate- } \\
\text { rial de transporte. Instalación y reparación industrial }\end{array}$ & $-12,03$ & $-2,17$ & 14,20 \\
\hline Construcción & $-11,71$ & 2,03 & 9,69 \\
\hline $\begin{array}{l}\text { Comercio al por mayor y al por menor y sus insta- } \\
\text { laciones y reparaciones. Reparación de automóviles, } \\
\text { hostelería }\end{array}$ & $-7,28$ & 1,18 & 6,10 \\
\hline $\begin{array}{l}\text { Transporte y almacenamiento. Información y comu- } \\
\text { nicaciones }\end{array}$ & $-11,61$ & $-5,44$ & 17,05 \\
\hline $\begin{array}{l}\text { Intermediación financiera, seguros, actividades in- } \\
\text { mobiliarias, servicios profesionales, científicos, ad- } \\
\text { ministrativos y otros }\end{array}$ & $-2,44$ & $-3,72$ & 6,15 \\
\hline $\begin{array}{l}\text { Administración Pública, educación y actividades sa- } \\
\text { nitarias }\end{array}$ & $-5,45$ & $-0,35$ & 5,81 \\
\hline Otros servicios & $-6,50$ & 2,01 & 4,49 \\
\hline Total & $-9,77$ & $-0,15$ & 9,92 \\
\hline
\end{tabular}

FUENTE: Elaboración propia a partir de microdatos de Encuesta de Población Activa.

más acusado han sido las industriales (salvo la de la industria de la alimentación, textil, cuero, madera y papel), la construcción y, dentro de los servicios, la rama de transportes y almacenamiento, información y comunicaciones. Entre las ramas donde se ha producido un menor trasvase destaca la de la agricultura, ganadería, silvicultura y pesca.

Los resultados parecen confirmar, por tanto, las dos hipótesis planteadas al inicio del artículo. Por un lado, en España se ha producido un proceso de destrucción de empleo más intenso en las actividades con una mayor intensidad de innovación tecnológica (proxy, en este trabajo, del nivel de automatización). Por otro lado, se observa que la demanda relativa de trabajadores más formados aumenta de forma más importante en las actividades con una intensidad de innovación tecnológica media o alta. 
En todo caso, el análisis presentado resulta descriptivo y tiene una serie de limitaciones que recomiendan una lectura prudente de los resultados. En primer lugar, un estudio pormenorizado de los efectos de la automatización requeriría disponer de información a nivel individual acerca de las tareas concretas realizadas por cada trabajador, así como de sus características, trayectoria laboral y las características de las empresas en las que desarrollan su empleo. Por otro lado, la variable que aproxima «automatización», la intensidad de innovación tecnológica, resulta una proxy imperfecta, al poder estar englobando innovaciones distintas a la automatización. Finalmente, debe considerarse que el período temporal analizado se corresponde solamente con las primeras etapas de la cuarta revolución industrial, cuyos efectos plenos posiblemente no podrán ser investigados hasta dentro de unos lustros.

\section{Conclusiones}

La robotización e introducción de inteligencia artificial en los procesos de producción plantean importantes retos en el mercado laboral. En este artículo se ha realizado una primera aproximación a dos de sus potenciales efectos: el efecto sobre el nivel de ocupación y sobre el tipo de trabajadores requeridos con la cuarta revolución industrial. A pesar de que el análisis realizado presenta limitaciones, los resultados parecen apuntar al hecho de que España no resulta una excepción a nivel internacional: durante la última década se ha producido una mayor reducción en el volumen de empleo en las actividades con una mayor intensidad de innovación tecnológica. Por el contrario, las actividades con una menor intensidad innovadora tecnológica han experimentado una menor reducción en la ocupación. En todas ellas, no obstante, se ha producido un cambio en la demanda del perfil formativo de los trabajadores. En todos los sectores se produjo un proceso de sustitución de trabajadores con una reducida dotación educativa por otros con estudios superiores. Ello resulta especialmente cierto en actividades con intensidad de innovación tecnológica media o alta. En el caso español, en los sectores de servicios y construcción también se observa una sustitución de trabajo poco cualificado por trabajo cualificado, si bien también actúa como refugio para trabajadores con niveles de formación intermedios. En este sentido, debe recordarse que el sector servicios engloba actividades muy dispares.

A la luz de lo anterior, para el caso español, la automatización de los procesos productivos parece, efectivamente, tener un efecto destructor de trabajo en los sectores más innovadores. Esta destrucción de empleo queda parcialmente compensada por trabajo creado en otros sectores, si bien la información con la que se ha trabajado no permite separar el efecto del ciclo económico del de la introducción de innovaciones.

Se abre con este artículo una vía de investigación que, en futuras etapas, debería profundizar en los efectos concretos sobre el empleo para cada actividad y por tipos de empresa. Los estudios venideros deberán considerar, a su vez, no solo el efecto 
de la automatización -y de tipos concretos de automatización- sobre el volumen de empleo, sino también sobre los salarios relativos de los trabajadores. Para ello, no obstante, resultará necesario disponer de datos más detallados que los disponibles en la actualidad para el caso español.

\section{Referencias bibliográficas}

[1] ACEMOGLU, D. y RESTREPO, P. (2017). «Robots and Jobs: Evidence from US Labor Markets». NBER Working Papers, 23285.

[2] AGHION, P.; JONES, B. F. y JONES, C. I. (2017). «Artificial Intelligence and Economic Growth». NBER Working Papers, 23928.

[3] ARNTZ, M.; GREGORY, T. y ZIERAHN, U. (2017). «Revisiting the Risk of Automation». Economics Letters, 159, 157-160.

[4] AUTOR, D. y SALOMONS, A. (2017). «Robocalypse Now: Does Productivity Growth Threaten Employment?». European Central Bank Sintra Forum Conference Paper, junio.

[5] BERG,A.; BUFFIE, E. y ZANNA, L. F. (2018). «Should We Fear the Robot Revolution? (The Correct Answer is Yes)». Journal of Monetary Economics.

[6] BRYNJOLFSSON, E. y MITCHELL, T. (2017). «What can machine learning do? Workforce implications». Science, 358 (6370), 1530-1534.

[7] BRYNJOLFSSON, E.; ROCK, D. y SYVERSON, C. (2017). «Artificial intelligence and the modern productivity paradox: A clash of expectations and statistics». NBER Working Papers, 24001.

[8] BUGHIN, J.; HAZAN, E.; RAMASWAMY, S.; CHUI, M.; ALLAS, T.; DAHLSTRÖM, P. y TRENCH, M. (2017). Artificial Intelligence-The Next Digital Frontier. McKinsey Glob Institute.

[9] CAINES, C.; HOFFMANN, F. y KAMBOUROV, G. (2017). «Complex-task biased technological change and the labor market». Review of Economic Dynamics, 25, 298319.

[10] CASELLI, F. y MANNING, A. (2018). «Robot arithmetic: new technology and wages». American Economic Review: Insights (en prensa).

[11] COWEN, T. (2018). «Neglected Open Questions in the Economics of Artificial Intelligence», en Economics of Artificial Intelligence. Chicago: University of Chicago Press (en prensa).

[12] CEDEFOP (2018). Insights into skill shortages and skill mismatch Learning from Cedefop's European skills and jobs survey. Luxembourg: Publications Office.

[13] COMISIÓN EUROPEA (2017). European Innovation Scoreboard 2017. Bruselas: Comisión Europea.

[14] DAUTH, W.; FINDEISEN, S.; SÜDEKUM, J. y WÖSSNER, N. (2017). «German robots: The impact of industrial robots on workers». IAB-Discussion Paper, 30/2017.

[15] DECKER, M.; FISCHER, M. y OTT, I. (2017). «Service Robotics and Human Labor: A first technology assessment of substitution and cooperation». Robotics and Autonomous Systems, 87, 348-354.

[16] DEKKER, F.; SALOMONS, A. y WAAL, J. V. D. (2017). «Fear of robots at work: the role of economic self-interest». Socio-Economic Review, 15(3), 539-562. 
[17] EICHHORST, W. (2015). «Do we have to be afraid of the future world of work? IZA Policy Paper, 102.

[18] FREY, C. B. y OSBORNE, M.A. (2017). «The Future of Employment: How Susceptible Are Jobs to Computerisation?». Technological Forecasting and Social Change, 114, 254-80.

[19] EICHHORST, W.; HINTE, H.; RINNE, U. y TOBSCH, V. (2017). «How big is the gig? Assessing the preliminary evidence on the effects of digitalization on the labor market». Management Revue, 28 (3), 298-318.

[20] GIBBS, M. (2017). «How is new technology changing job design?». IZA World of Labor, 2017: 344.

[21] HUTTER, C. y WEBER, E. (2017). «The effects of skill-biased technical change on productivity flattening and hours worked». IAB-Discussion Paper, 32/2017.

[22] KORINEK, A. y STIGLITZ, J. E. (2017). «Artificial intelligence and its implications for income distribution and unemployment». NBER Working Papers, 24174.

[23] LEVY, F. (2018). «Computers and populism: artificial intelligence, jobs, and politics in the near term». Oxford Review of Economic Policy, 34 (3), 393-417.

[24] MALONEY, W. F. y MOLINA, C. A. (2016). Are automation and trade polarizing developing country labor markets, too? World Bank Policy Research WP, 7922.

[25] MORIKAWA, M. (2017). «Who are afraid of losing their jobs to artificial intelligence and robots? Evidence from a survey». GLO Discussion Paper, 71.

[26] MOKYR, J. (2018). «The past and the future of innovation: Some lessons from economic history». Explorations in Economic History, 69.

[27] SACHS, J. D.; BENZELL, S. G. y LAGARDA, G. (2015). «Robots: Curse or Blessing? A Basic Framework». NBER Working Papers, 21091.

[28] RAJ, M. y SEAMANS, R. (2018). «AI, Labor, Productivity, and the Need for FirmLevel Data», en Economics of Artificial Intelligence. Chicago: University of Chicago Press (en prensa).

[29] SCHMIDPETER, B. y WINTER-EBMER, R. (2018). «How Do Automation and Offshorability Influence Unemployment Duration and Subsequent Job Quality?». Discussion Paper, 11736. Institute for the Study of Labor (IZA).

[30] VARIAN, H. (2018). «Artificial intelligence, economics, and industrial organization», en The Economics of Artificial Intelligence: An Agenda. University of Chicago Press (en prensa).

[31] VIVARELLI, M. (2014). «Innovation, Employment and Skills in Advanced and Developing Countries: A Survey of Economic Literature». Journal of Economic Issues, 48 (1), 123-54. 


\section{ANEXO \\ TABLA A.1 \\ CLASIFICACIÓN DE ACTIVIDADES EN FUNCIÓN DEL ÍNDICE DE INTENSIDAD DE INNOVACIÓN}

Intensidad de innovación baja (índice $<\mathbf{0 , 4}$ )

1. AGRICULTURA, GANADERÍA, SILVICULTURA Y PESCA

2.2. Industrias del petróleo (CNAE 19)

20. Energía y agua (CNAE 35, 36)

21. Saneamiento, gestión de residuos y descontaminación (CNAE 37, 38, 39)

22. Construcción (CNAE 41, 42, 43)

23. Comercio (CNAE 45, 46, 47)

25. Hostelería (CNAE 55, 56)

28. Actividades inmobiliarias (CNAE 68)

30. Actividades administrativas y servicios auxiliares (CNAE 77, 78, 79, 80, 81, 82)

31. Actividades sanitarias y de servicios sociales (CNAE 86, 87, 88)

32. Actividades artísticas, recreativas y de entretenimiento (CNAE 90, 91, 92, 93)

Intensidad de innovación media (índice entre 0,4 y 1)

3. Alimentación, bebidas y tabaco (CNAE 10, 11, 12)

4.1. Textil (CNAE 13)

4.3. Cuero y calzado (CNAE 15)

5. Madera, papel y artes gráficas (CNAE $16,17,18)$

5.1. Madera y corcho (CNAE 16)

5.2. Cartón y papel (CNAE 17)

5.3. Artes gráficas y reproducción (CNAE 18)

9. Productos minerales no metálicos diversos (CNAE 23)

10. Metalurgia (CNAE 24)

17. Muebles (CNAE 31)

19. Reparación e instalación de maquinaria y equipo (CNAE 33)

24. Transportes y almacenamiento (CNAE 49, 50, 51, 52, 53)

27. Actividades financieras y de seguros (CNAE 64, 65, 66)

33. Otros servicios (CNAE 85-854, 94, 95, 96)

Intensidad de innovación alta (índice >1)

2.1. Industrias extractivas (CNAE 05, 06, 07, 08, 09)

4. Textil, confección, cuero y calzado (CNAE 13, 14, 15)

4.2. Confección (CNAE 14)

6. Química (CNAE 20)

7. Farmacia (CNAE 21)

8. Caucho y plásticos (CNAE 22)

11. Manufacturas metálicas (CNAE 25)

12. Productos informáticos, electrónicos y ópticos (CNAE 26)

13. Material y equipo eléctrico (CNAE 27) 
TABLA A.1 (Cont.)

CLASIFICACIÓN DE ACTIVIDADES EN FUNCIÓN DEL ÍNDICE DE INTENSIDAD DE INNOVACIÓN

14. Otra maquinaria y equipo (CNAE 28)

15. Vehículos de motor (CNAE 29)

16. Otro material de transporte (CNAE 30)

16.1. Construcción naval (CNAE 301)

16.2. Construcción aeronáutica y espacial (CNAE 303)

16.3. Otro equipo de transporte (CNAE 30-301-303)

18. Otras actividades de fabricación (CNAE 32)

26. Información y comunicaciones (CNAE 58, 59, 60, 61, 62, 63)

26.1. Telecomunicaciones (CNAE 61)

26.2. Programación, consultoría y otras actividades informáticas (CNAE 62)

26.3. Otros servicios de información y comunicaciones (CNAE 58, 59, 60, 63)

29. Actividades profesionales, científicas y técnicas (CNAE 69, 70, 71, 72, 73, 74, 75)

29.1. Servicios de I+D (CNAE 72)

29.2. Otras actividades (CNAE 69, 70, 71, 73, 74, 75) 
TABLA A.2

DISTRIBUCIÓN DE LOS OCUPADOS SEGÚN SU NIVEL EDUCATIVO Y RAMA DE ACTIVIDAD. 2007 (SEGUNDO TRIMESTRE). ESPAÑA (En \%)

\begin{tabular}{|c|c|c|c|}
\hline & $\begin{array}{l}\text { Hasta } \\
\text { secundaria } \\
\text { inferior }\end{array}$ & $\begin{array}{l}\text { Secundaria } \\
\text { superior }\end{array}$ & Superior \\
\hline Agricultura, ganadería, silvicultura y pesca & 73,54 & 16,46 & 10,00 \\
\hline $\begin{array}{l}\text { Industria de la alimentación, textil, cuero, madera y } \\
\text { papel }\end{array}$ & 56,06 & 21,51 & 22,43 \\
\hline $\begin{array}{l}\text { Industrias extractivas, refino de petróleo, industria } \\
\text { química, farmacéutica, industria del caucho y mate- } \\
\text { rias plásticas, suministro energía eléctrica, gas, vapor } \\
\text { y aire acondicionado, suministro de agua, gestión de } \\
\text { residuos. Metalurgia }\end{array}$ & 47,78 & 22,71 & 29,51 \\
\hline $\begin{array}{l}\text { Construcción de maquinaria, equipo eléctrico y mate- } \\
\text { rial de transporte. Instalación y reparación industrial }\end{array}$ & 41,31 & 25,30 & 33,39 \\
\hline Construcción & 65,46 & 19,58 & 14,97 \\
\hline $\begin{array}{l}\text { Comercio al por mayor y al por menor y sus insta- } \\
\text { laciones y reparaciones. Reparación de automóviles, } \\
\text { hostelería }\end{array}$ & 50,38 & 28,66 & 20,96 \\
\hline $\begin{array}{l}\text { Transporte y almacenamiento. Información y comu- } \\
\text { nicaciones }\end{array}$ & 41,17 & 29,06 & 29,76 \\
\hline $\begin{array}{l}\text { Intermediación financiera, seguros, actividades in- } \\
\text { mobiliarias, servicios profesionales, científicos, ad- } \\
\text { ministrativos y otros }\end{array}$ & 24,14 & 23,18 & 52,68 \\
\hline $\begin{array}{l}\text { Administración Pública, educación y actividades } \\
\text { sanitarias }\end{array}$ & 17,11 & 20,59 & 62,29 \\
\hline Otros servicios & 48,42 & 28,08 & 23,50 \\
\hline Total & 43,34 & 23,89 & 32,77 \\
\hline
\end{tabular}

FUENTE: Elaboración propia a partir de microdatos de Encuesta de Población Activa (INE). 